\title{
Successful treatment of normocomplementemic urticarial vasculitis with omalizumab: A report of three cases and literature review
}

\author{
Teerapong Rattananukrom, ${ }^{1}$ Pranee Svetvilas, ${ }^{1}$ Kumutnart Chanprapaph ${ }^{1}$
}

\begin{abstract}
Urticarial vasculitis (UV) is a rare form of cutaneous leukocytoclastic vasculitis with persistent urticarial lesions. UV may be severe and refractory to standard treatment including antihistamines, anti-inflammatories, antimalarials, corticosteroids and immunosuppressants. Omalizumab, an anti-IgE antibody, is approved for chronic spontaneous urticaria. However, its benefit for UV remains controversial. We report, herein, three patients with normocomplementemic UV and angioedema. All patients were diagnosed with chronic urticaria preceding the presentation of painful urticarial plaques. The diagnosis of UV was confirmed by skin biopsy and/or direct immunofluorescence. All patients had none or minimal response to standard treatments. Initial omalizumab dosing of $150 \mathrm{mg}$ was administered subcutaneously (SC), however, increment to $300 \mathrm{mg}$ monthly was necessary in 2 patients to control the disease. All 3 patients remained in complete remission after minimum follow up period of 9 months. To conclude, omalizumab has shown to be beneficial for severe normocomplementemic UV in our series.
\end{abstract}

Key words: Omalizumab, Urticarial vasculitis, Leukocytoclastic vasculitis, Urticaria, Antihistamine

From:

Division of Dermatology, Department of Internal Medicine, Faculty of Medicine, Ramathibodi Hospital, Mahidol University, Bangkok, Thailand

\section{Introduction}

Urticarial vasculitis (UV) is a rare type of cutaneous leukocytoclastic vasculitis characterized by painful wheal and flare persisting more than 24 hours. Histopathologically, UV demonstrates superficial perivascular infiltration with leukocytoclasia. UV is categorized into normocomplementemic with skin-limited disease and hypocomplementemic typically with systemic involvement (e.g. fever, arthralgia, uveitis, glomerulonephritis, abdominal pain). UV is mostly idiopathic but can be related to other autoimmune diseases, chronic infection, drugs or malignancy. ${ }^{1}$

Treatment for UV comprise of antihistamines, anti-inflammatories, antimalarials, corticosteroids and immunosuppressant. ${ }^{1}$ Severe cases may not respond and/or experience adverse effects from standard modalities. Omalizumab is FDA-approved for chronic spontaneous urticaria (CSU), but currently has limited reports for the treatment of UV., ${ }^{2,3}$ We report three cases of normocomplementemic UV successfully treated and controlled with omalizumab.

\section{Corresponding author:}

Kumutnart Chanprapaph

Division of Dermatology, Faculty of Medicine Ramathibodi Hospital, Mahidol University

270 Rama VI Road, Rachathewi, Bangkok, Thailand 10400

E-mail: kumutnartp@hotmail.com

\section{Case Reports \\ Case 1}

A 45-year-old woman with allergic rhinitis and nasal squamous carcinoma presented with chronic urticaria and angioedema. Fexofenadine and desloratadine were titrated to maximum dosage without improvement. Oral prednisolone $(0.5 \mathrm{mg} / \mathrm{kg} /$ day $)$ was used intermittently to control exacerbation. She later developed multiple, erythematous edematous plaques with hyperpigmentation predominantly on the extremities. Skin biopsy demonstrated leukocytoclastic vasculitis. Direct immunofluorescence (DIF) was negative. Laboratory investigations demonstrated positive antinuclear antibodies (ANA, anticentromere; 1:1280) but other systemic lupus erythematosus (SLE)-associated serology were negative (anti-Smith (Sm), anti-ribonucleoproteins (RNP) and anti-double stranded DNA (anti-dsDNA). Anti-HIV, antithyroglobulin (anti-TG), anti-thyroid peroxidase (antiTPO), and hepatitis $\mathrm{B}$ and $\mathrm{C}$ were negative. Complete blood count $(\mathrm{CBC})$ and complement proteins (C3 $1.42 \mathrm{~g} / \mathrm{L}$, normal range (NR) $0.9-1.8, \mathrm{C} 40.19 \mathrm{~g} / \mathrm{L}, \mathrm{NR} 0.1-0.4)$ were normal. 
Erythrocyte sedimentation rate (ESR) was $22 \mathrm{~mm} / \mathrm{hr}$ (NR 4-20). The diagnosis of normocomplementemic UV was made. Hydroxychloroquine (200 mg/day), colchicine $(1.2 \mathrm{mg} /$ day), montelukast (10 mg/day), indomethacin (50 mg/day) and dapsone (100 $\mathrm{mg} /$ day) provided insufficient response. Omalizumab (150 mg SC) was administered as an alternative treatment. Weekly urticaria activity score (UAS7) prior to omalizumab injection was 42. After 4 weeks, the rash improved partially (UAS7 of 37), omalizumab was increased to $300 \mathrm{mg}$ SC monthly. Complete remission was achieved at week 20 (UAS7 of 0 ). Following six consecutive treatments, omalizumab was spaced to every 6-8 weeks, for a total of 10 injections. She remained asymptomatic throughout the oneyear follow-up. Other than continuous injection of omaluzumab, all previous treatments were discontinued.

\section{Case 2}

A 50-year-old previously healthy man presented with urticaria and angioedema for 2 months. He had positive ANA (coarse speckled; 1:320) but was negative for other SLE-associated serology. Anti-TG, anti-TPO, hepatitis B and C were negative. $\mathrm{CBC}$ and stool exam were normal. The patient responded partially to levocetirizine titrated up to fourfolds. Two months later, he developed persistent painful annular erythematous edematous plaques on the back. Skin biopsy showed neutrophilic infiltration surrounding superficial blood vessels with leukocytoclasia. DIF was non-specific. His complement levels (C3 $1.28 \mathrm{~g} / \mathrm{L}$, C4 $0.29 \mathrm{~g} / \mathrm{L}$ ) and ESR (3 mm/hr) were normal. Normocomplementemic UV was diagnosed (UAS7 of 21). Oral prednisolone (10 $\mathrm{mg} /$ day), desloratadine (20 mg/day), colchicine (0.6 mg/day),

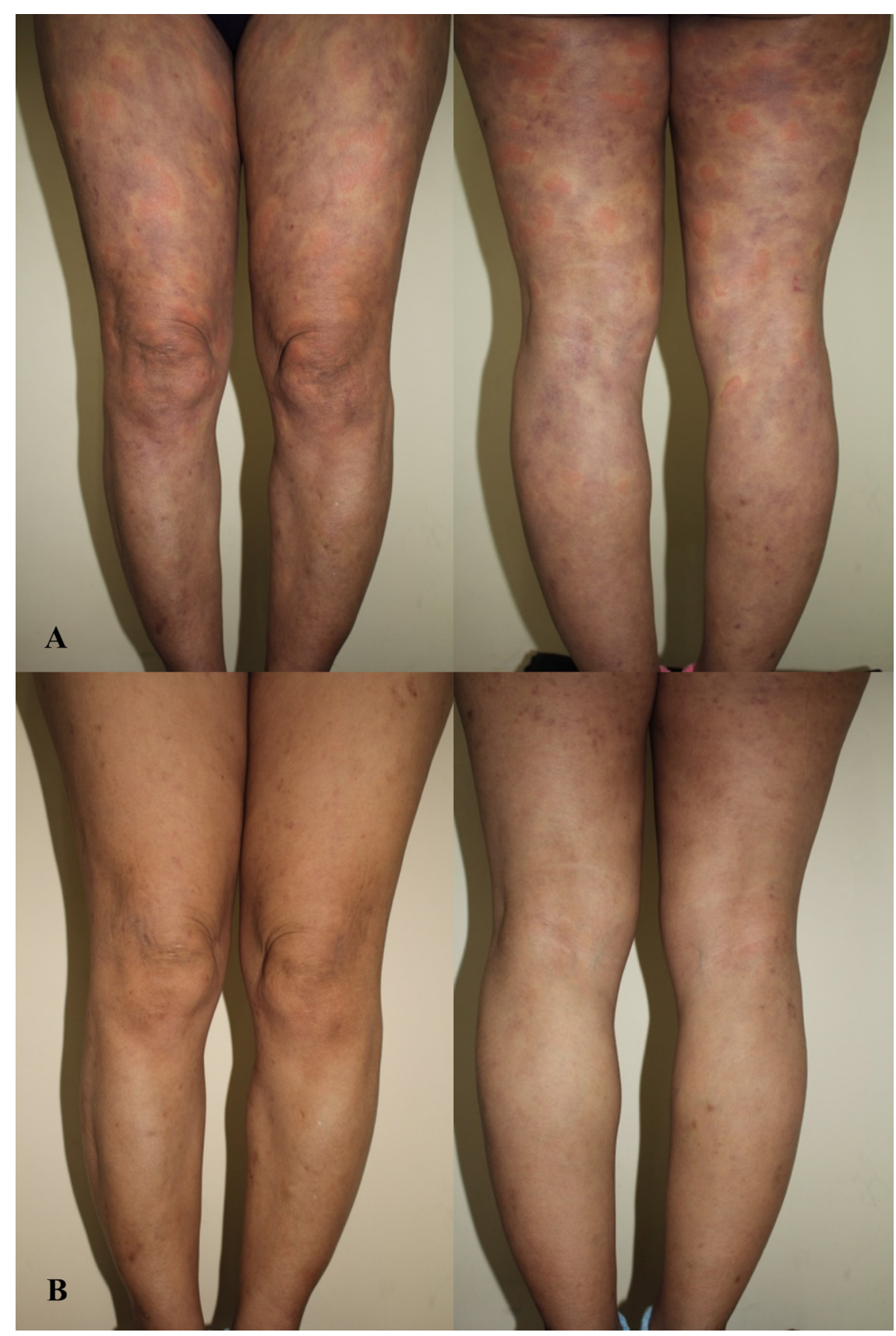

Figure 1. A 51-year-old female experiencing (A) painful erythematous edematous plaques with hyperpigmentation (B) resolution after 2 weeks of omalizumab $(300 \mathrm{mg}, \mathrm{SC})$ 


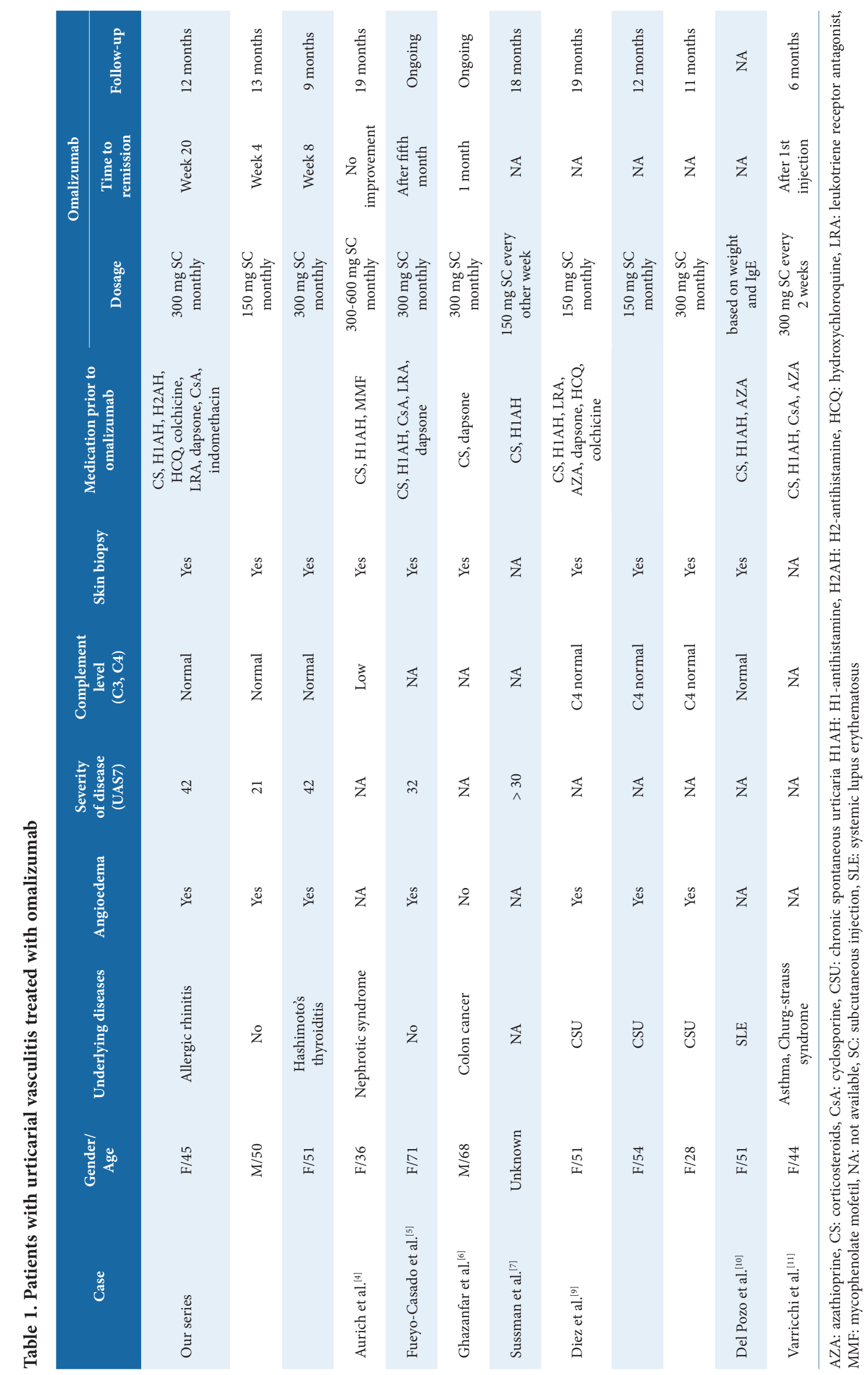


and indomethacin (50 mg/day) gave inadequate response. Cyclosporine $(1.5 \mathrm{mg} / \mathrm{kg} /$ day) was commenced but later discontinued due to the deterioration of renal function. The first omalizumab (150 mg) was injected. After four weeks he had remission (UAS7 of 0 ). Omalizumab was commence every 4 weeks, then the interval of injection was increased to every 6 weeks for a total of 12 injections. Prior therapies with the exception of omalizumab was discharged. The patient remained asymptomatic throughout the 13-month follow-up.

\section{Case 3}

A 51-year-old woman diagnosed with CSU and angioedema for 2 years. Fexofenadine and desloratadine were titrated to maximum dosage without response. Oral prednisolone $(0.5 \mathrm{mg} / \mathrm{kg} /$ day $)$ was commenced intermittently. Montelukast (10 mg/day), dapsone (50 mg/day) cyclosporine $(1.5 \mathrm{mg} / \mathrm{kg} /$ day) was ineffective. A month later, she developed painful erythematous edematous plaques with hyperpigmentation on the trunk and extremities (Figure 1). Skin biopsy showed perivascular mixed cells inflammation without leukocytoclasia. DIF showed granular IgM and C3 deposition along the superficial blood vessels, compatible with small vessel vasculitis. Laboratory investigations showed positive ANA (anticentromere titer; $1: 1280$ ) and anti-TPO of $>4,000 \mathrm{U} / \mathrm{ml}$, while other SLE-associated laboratories were negative and TSH was normal. Anti-HIV, hepatitis B and C, CBC and complements (C3 $1.13 \mathrm{~g} / \mathrm{L}, \mathrm{C} 40.16 \mathrm{~g} / \mathrm{L}$ ) were normal. ESR was $40 \mathrm{~mm} / \mathrm{hr}$. The diagnosis of normocomplementemic UV was made (UAS7 of 42). Omalizumab (150 mg SC) prescribed initially gave partial response. Dose increment to $300 \mathrm{mg}$ commenced a month later offered dramatic response within 2 weeks (Figure 1b). Complete remission was achieved at week eight (UAS7 of 0). Ongoing treatment with omalizumab 300 $\mathrm{mg} / \mathrm{month}$ gave sustained response at nine months follow-up. She has been on continuous treatment with only omalizumab thereafter.

\section{Discussion}

UV is an immune complex mediated disease with complement activation, increase capillary permeability and upregulation of neutrophil chemotaxis. ${ }^{5}$ Therapy for severe UV can be challenging as patients may response poorly to standard regimen. Moreover, long-term maintenance with systemic corticosteroid and immunosuppressants (i.e. cyclosporine) is not desirable nor acceptable.

Omalizumab, a humanized anti-IgE monocloncal antibody, produces several well-recognized immunomodulatory effects for CSU i.e. sequestering monomeric IgE, down regulates FCeR1 on mast cells and basophils and reduces B-cell activation and homing. ${ }^{2}$ Its benefit for UV remains unclear but may share the same mechanism. While there are no prospective studies of omalizumab for UV, few case reports have shown favorable outcome for this indication (Table 1).

We report three patients with normocomplementemic UV with angioedema confirmed by skin biopsy and/or DIF which failed all previous therapies. All patients lacked sufficient clinical and immunological criteria for the diagnosis of SLE. UAS, a validated tool to assess severity for CSU (not UV), was used prior to first injection and throughout follow-up period.
Omalizumab was administered at dosage of $150 \mathrm{mg}$, and 300 mg monthly, as per pervious recommendations..$^{5-7}$ Optimum initial dosage of omalizumab for UV is uncertain. Our patients achieved clinical remission (UAS7 of 0 ) at 20,4 , and 8 weeks after omalizumab, respectively. None experienced adverse reaction. We attempted to prolong the dosing interval for our patients at 6-months, where it was extended to every 6-8 weeks. All of our patients were able to discontinue prior medications entirely. Although stepwise management guideline for symptom control of CSU is very clear, there is currently no evidence on how to perform omalizumab discontinuation. Some authors reduce monthly dosage and some lengthens the interval to 6-8 weeks or even 2-3 months. ${ }^{5-8}$

There are currently nine reported cases of omalizumab for

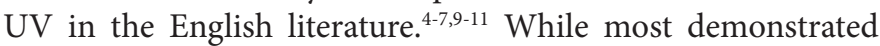
satisfactory outcomes, Aurich et al. described no improvement for hypocomplementemic UV. ${ }^{4}$ We propose that omalizumab may have a promising role for severe normocomplementemic UV. Nevertheless, accumulation of more cases and clinical trials to compare standard treatment with omalizum$\mathrm{ab}$ in UV are necessary.

In conclusion, we report successful treatment with omalizumab for normocomplementemic UV. Although, the mechanism of omalizumab for UV remains unclear, great benefits have been demonstrated for our UV patients unresponsive to standard modalities.

\section{Conflict of interest}

The authors have none to declare

\section{References}

1. Venzor J, Lee WL, Huston DP. Urticarial vasculitis. Clin Rev Allergy Immunol. 2002;23:201-16.

2. Oliver JM, Tarleton CA, Gilmartin L, Archibeque T, Qualls CR, Diehl $\mathrm{L}$, et al. Reduced FcepsilonRI-mediated release of asthma-promoting cytokines and chemokines from human basophils during omalizumab therapy. Int Arch Allergy Immunol. 2010;151:275-84.

3. Urgert MC, van den Elzen MT, Knulst AC, Fedorowicz Z, van Zuuren EJ. Omalizumab in patients with chronic spontaneous urticaria: a systematic review and GRADE assessment. Br J Dermatol. 2015;173:404-15.

4. Aurich S, Simon JC, Treudler R. Omalizumab does not improve skin lesions in a patient with hypocomplementemic urticarial vasculitis syndrome. J Eur Acad Dermatol Venereol. 2017;31:e395-7.

5. Fueyo-Casado A, Campos-Munoz L, Gonzalez-Guerra E, Pedraz-Munoz J, Cortes-Toro JA, Lopez-Bran E. Effectiveness of omalizumab in a case of urticarial vasculitis. Clin Exp Dermatol. 2017;42:403-05.

6. Ghazanfar MN, Thomsen SF. Omalizumab for Urticarial Vasculitis: Case Report and Review of the Literature. Case Rep Dermatol Med. 2015;2015:576893.

7. Sussman G, Hébert J, Barron C, Bian J, Caron-Guay RM, Laflamme S, et al. Real-life experiences with omalizumab for the treatment of chronic urticaria. Ann Allergy Asthma Immunol. 2014;112:170-4.

8. Larenas-Linnemann DES, Parisi CAS, Ritchie C, Cardona-Villa R, Cherrez-Ojeda I, Cherrez A, et al. Update on Omalizumab for Urticaria: What's New in the Literature from Mechanisms to Clinic. Curr Allergy Asthma Rep. 2018;18:33.

9. Díez LS, Tamayo LM, Cardona R. [Omalizumab: therapeutic option in chronic spontaneous urticaria difficult to control with associated vasculitis, report of three cases]. Biomedica. 2013;33:503-12. Spanish.

10. Ramírez del Pozo ME, Martínez-Saenz NP, Vera JG, Tiro JL. 274 Vasculitic Urticaria Treated with Omalizumab. Case Report. World Allergy Organ J. 2012;5 Suppl 2:S106.

11. Varricchi G, Detoraki A, Liccardo B, Spadaro G, de Paulis A, Marone G, et al. 275 Efficacy of Omalizumab in the Treatment of Urticaria -Vasculitis Associated to Churg-Strauss Syndrome: A Case Report. World Allergy Organ J. 2012;5 Suppl 2:S106-7. 